\title{
Aperture Filter and Adaptive Filter Response for Reconstruction of Sea Surface Image Based on LIDAR Observation
}

\author{
Muhammad Sameer Sheikh, QunSheng Cao, and Caiyun Wang
}

\begin{abstract}
This paper discusses the reconstruction of seasurface photo imaging based on Light Detection and Ranging (LIDAR) scanning observation, the new framework consists of three phases. Firstly, we set the angles and range of the image to perform the scanning to get the pixel coordinates of seasurface image in $x$ and $y$ direction respectively. Secondly, we get the continuous signal and sampled by using aperture filter and combine the patches into the piecewise form to achieved the smoothly reconstruction. Finally, we applied the adaptive filter to increases the resolution of the image and to filter out the noise present in the image. The proposed method demonstrates the better reconstruction of sea-surface image. In addition we performed error simulation and the noise evaluation of the aperture filter, and it is noted that the error is significantly reduced compared with other method.
\end{abstract}

Index Terms-Adaptive filter, aperture filter, flash LIDAR, pixel reconstruction, photo imaging, sea surface image.

\section{INTRODUCTION}

The problem occurs of non-uniform sampling, and reconstruction has been received the considerable attention from the researchers in the past decades, indeed the sampling and reconstruction for a band limited signal when there is no reconstruction of the distribution of sampling [1]. The technology of the Light Detection and Ranging (LIDAR) refers to a radar system operating at optical frequencies that uses a laser as a photon source, it has found great application, likewise, geology, geography and so on [2], [3] and is not done by using that application in a photo image. The solution of the problem of reconstruction of photo image ocean surface which is based on the concept of LIDAR scanning mechanism and adaptive filter method is very vital in real application. We have seen that the previous work has been done in biomedical application using the line integrals of real projection data to reconstruct the discrete image [4], and some of the algorithms have been proposed to reconstruct the radiometric image by integrating each pixel over the time [5], but this technique work efficiently by defining the higher temperature level.

The reconstruction of photo imaging ocean surface is very

Manuscript received September 16, 2015; revised November 2, 2015. Muhammad Sameer Sheikh and Qunsheng Cao are with College of Electronics and Information Engineering, Nanjing University of Aeronautics and Astronautics. Nanjing, China (e-mail: sameer.5@hotmail.com).

Caiyun Wang is with College of Astronautics Nanjing University of Aeronautics and Astronautics, Nanjing 210016, China (e-mail: wangcaiyun@nuaa.edu.cn). different in previous approaches, some reconstructions using filtered back projection were depended on the geometrical view of the image [4]. And some methods using integrated each pixel corresponding to the time and likewise, the qualities of the image only were depended on the time respectively [5]. Reconstruction of sea image have been used based on sparse method which is famous technique but require large amount of calculation [6]. And some methods were used only to suppress an additive noise in a continuous image sequence by adaptive filtering method [7]. In the past, the synthetic aperture radar (SAR) imaging using randomly sampled data to reconstruct the image based on the compressed sensing (CS) theory, which is the more common and efficient method for sensing signals used [8]. Once getting the reconstruction image, the resolution would be the main problem, and normally, it could not obtain the highresolution image [9] had forward to over sampling method to overcome this problem [9], it did by the scanning mechanism with different values to achieve the highest resolution reconstructed image [10], figured out the ocean waves and developed method to evaluate the behavior by using Flash LIDAR.

Some reconstructions of the image by scatterometer imaging method have been defined but there are some restrictions to a reconstruction operator [11]. In addition, some work in past decades considered to provide the resolution of image by only the multiple frame of the low resolution, noisy image which has some limitations because of the frames contain noises [12]. Band limited reconstruction by using multidimensional was depended on sine function involve by using Cartesian geometry, which is done by irregular sampling [13]. There were some limitations when it was applied the Aerial Laser Scanning it was restricted to the surface modeling [14], and some of the methods previously done, likewise, the reconstruction of the CT images were based on the Shearlet transformation, which has been mainly applied in biomedical imaging but causes artifacts [15]. In some cases solution presented in the LMS algorithm to provide the higher resolution has some restriction [16]. And it was used adaptive filter for image resolution by defining with LIDAR scanning mechanism, and some method is depended on the selection of the operator to do the reconstruction of the image [17].

Our objective is to get reconstructed photo imaging of ocean surface by using Aperture Filter sampling techniques by scanning mechanism of LIDAR and then process by an adaptive filter to enhance the better reconstructed image, finally the model described for image reconstruction is simple and exactly reconstruction. We adopt the Flash 
LIDAR scanning in two-dimensional (2D) case to achieve the maximum information about the image in order to reconstruct. Once we get the signal, we use aperture filtering sampling method to achieve the exact sampling of the signal, and after we reconstruct the pixel by smoothing kernel techniques in order to make it sharper and smooth, then after we apply the adaptive filter to an image to get the best resolution of the ocean surface image.

This paper is organized as follows. In Section II, we discuss about the modeling of the scheme and analysis as well as the patch selection. Section III presents the pixel reconstruction and resolution methodology. We perform the adaptive filtering in Section IV. In Section V we discuss about noise modeling of the aperture filtered samples. Section VI we discusses the simulation results and finally Section VII concludes the paper.

\section{SCHEME MODELING AND ANALYSIS}

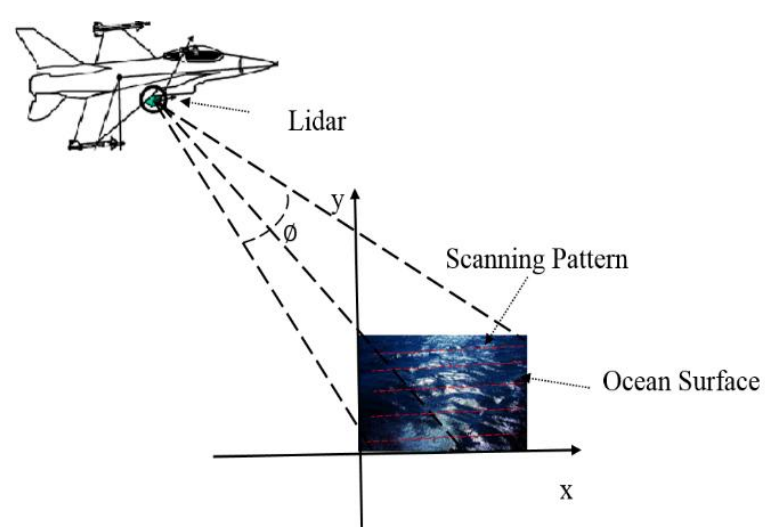

Fig. 1. Geometrical view.

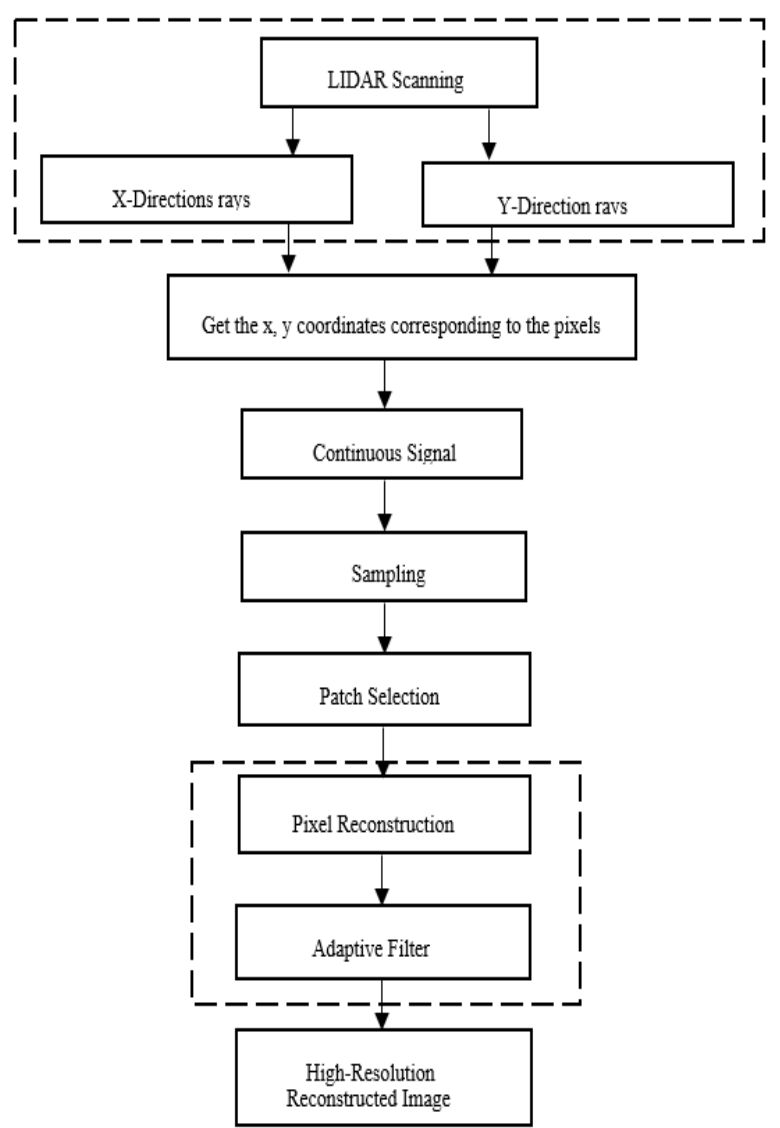

Fig. 2. Process of block diagram.

Fig. 1 is the geometrical view of the proposed model. It is assumed the LIDAR is assembled in the plane which is in the forward direction, above the sea level and the scanning parameters have been defined before a scan. The angle of the scanning is defined as $\Phi, N_{x}$ and $N_{y}$ are the number of the coordinates corresponding to the pixels along in the horizontal and the vertical directions, respectively. The scanning plane is performed in $2 \mathrm{D}$ case, and the block diagram is shown in Fig. 2.

In Fig. 2, we begin performing the LIDAR scanning by setup an angle and defining parameters in both $x$ and $y$ directions. An image is taken and performed the scanning by every point of the scanning to obtain the coordinates of the image corresponding to the pixel by using the MATLAB tool. Once the coordinates $(x, y)$ of the images are achieved, it processes and converts the coordinates into the linear continuous signal form by defining and assuming parameters to get the signal in the continuous form. Our objective is not only the reconstruction of the ocean surface image, but also an emphasis on the high resolution photo image, so firstly we perform to sample the continuous signal by using the aperture filtering method, secondly we have defined the cubical geometry of the patch, which is very vital for the pixel reconstruction. In addition, we assumed that the reconstruction of the ocean surface is piecewise, and every patch does contain noise. From the noisy sample of the image, we apply adaptive filtering techniques corresponding to the LIDAR scanning to achieve the highest resolution reconstructed image

\section{A. LIDAR Scanning Modeling Mechanism}

The LIDAR is assumed to be assembled in the plane, and it performs scanning in the $x-y$ plane shown in Fig. 3. In order to make the efficient scanning pattern to get the maximum information of the ocean surface image, we start to scan the image by defining the parameter, and get the pixels of the each scanning point along the $x$ and $y$ directions to get the maximum information of the ocean surface image.

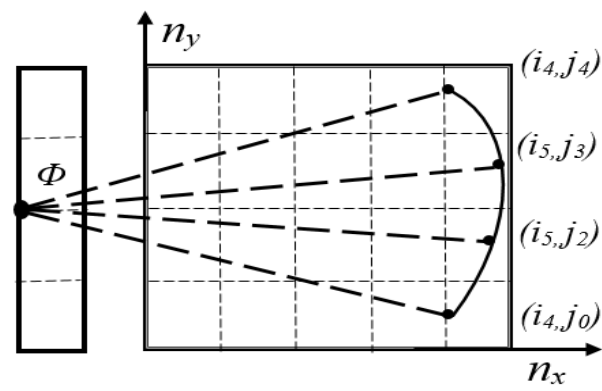

Fig. 3. LIDAR scanning pattern mechanism.

Fig. 3 is shown the general LIDAR scanning pattern. The scanning angle is $\Phi, n_{\mathrm{x}}$ and $n_{\mathrm{y}}$ are the number of pixels along $x$ - and $y$-direction, respectively. The image pixel of each coordinates $(x, y)$ is discretized into a point $(i, j)$ which is discussed in Section V. From Fig. 3, using the traditional technology of data transformation. The image pixel information is converted a continuous one-dimensional (1D) signal form by gathered all data in both directions. It is assumed the defined signal has the cosine and sine functions for conversion of the signal [10]. 


$$
n_{x}=\sum_{i=1}^{N_{x}}\left|k_{i}\right|^{-3 / 2}\left(x_{i} \cos \alpha_{i}\right)
$$

where $\alpha_{i}$ is the coefficient of the scanning mechanism along the $x$-direction and $k_{\mathrm{i}}$ is the constant parameter which creates harmonics amplitude. Similarly, the signal is obtained by scanning along $y$-direction.

$$
n_{y}=\sum_{j=1}^{N_{x}}\left|k_{j}\right|^{-3 / 2}\left(y_{j} \sin \beta_{j}\right)
$$

where $\beta_{j}$ is the scanning coefficient along $y$-direction. Combining Eqns. (1) and (2) into a continuous signal to form reconstruct signal, the appropriate response of continuous signal is as follows [9].

$$
n_{x y}=\sum_{i,=1}^{N_{x}} \sum_{j=1}^{N_{y}}\left|k_{i, j}\right|^{-3 / 2}\left(x_{i} \cos \alpha_{i}\right)\left(y_{j} \sin \beta_{j}\right)
$$

where $k_{i j}$ is the harmonic amplitude and the square root of the energy density spectrum, and $x_{i}$ and $y_{j}$ are the coordinates corresponding to the pixels in $x$ and $y$ directions, respectively. The coordinates corresponding to the pixel are converted into 1D linear expression.

The LIDAR scanning model in our design is based on the scanning rays, for all of the scannings we performed, we keep the LIDAR scanning rays is same for both $x$ and $y$ direction respectively, if we don't keep the same rays, scanning for both directions, so it might be possible that we may lose the information when reconstructed the image, the general rays scanning direction shown in Fig. 3, then it performed the scanning on the original image to get the coordinates of the image corresponding to the pixels.

$$
n_{x y}=\sum_{i,=1}^{N_{x}} \sum_{j=1}^{N_{y}}\left|k_{i, j}\right|^{-3 / 2} A_{i} B_{j}\left(\cos \alpha_{i}\right)\left(\sin \beta_{j}\right)
$$

In order to make it is easier to operate, $n_{x y}$ is replaced by the total reconstruction signal $S(x)$, that is,

$$
n_{x y}=S(x)
$$

Eq. 5 is the simplified form of the Eq. 4, which is representing the $1 \mathrm{D}$ continuous form of the signal.

\section{B. Sampling Theory and Methodology}

The sampling way is very vital to digital signal processing, some of the methods that have been applied to reconstruct the image from sparse data, but violate the Nyquist spatial sampling criteria [18]. It is widely known that the sampling theory is restricted to finite sample numbers, and thus the signal is band limited and continuous periodic. When a practical signal is reconstructed, it causes so the aliasing effect, the periodic signal is considered with a band limited, so aliasing effect is negligible in this case. In general, the aperture of the sampling performed is larger than the sampling space, and there is no cross talk or overlapping between the adjacent pixels. If the band limited by frequency $\omega_{0}$ is imposed by aperture function, every aperture function is band limited, thus the sampling performed on the continuous signal is same as the sampling performs on the band limited signal, otherwise, and higher bandwidth is used to minimize the aliasing effect [11]. The sampling produces sequences, which the samples represent the values of the original image signal. They are express as following:

$$
[S]=\left[\begin{array}{c}
S\left(x_{1}\right) \\
\cdot \\
\cdot \\
S\left(x_{N}\right)
\end{array}\right]=\left[\begin{array}{c}
\int A_{1} S_{1}(x) d x \\
\cdot \\
\cdot \\
\int A_{N} S_{N}(x) d x
\end{array}\right]=\int[A] S(x) d x
$$

where $[S]$ is represented the aperture filtered samples, which the samples represent the pixel values of the original signal, $N$ is the total number of the samples, $A$ is the sampling operator of the band limited signal and apply on the signal samples $S$ The discretization of the signal from the use of the aperture filtered sample is basic requirement of reconstruction to the ocean surface high-resolution image.

\section{Patching Selection and Transformation}

The patch is presented in an image as a bag of sampled patches on a regular grid, and each pixel is considered as a basic element of an image, editing of an image can be done shuffling of the patches on the image. The previous researches have been done by inverting the patches to reconstruct an image [19], [20], but this cause blurriness in an image.

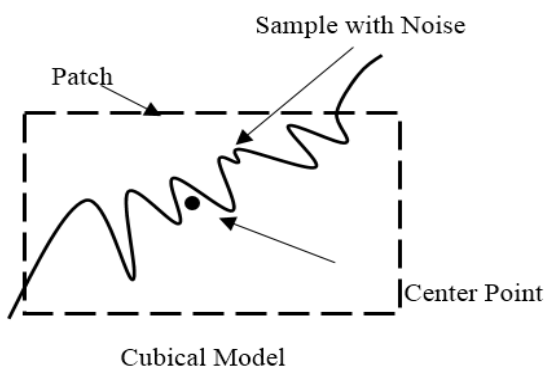

Fig. 4. Patch geometrical model.

Fig. 4 is the geometrical view of the patch of the constant window size is assumed, likewise, width and height of the patch, when we performed the reconstruction of the pixel based on the patch, each patch contains noisy samples. This noise effect of the samples presented in the patch is less than the conventional filter because the aperture filter has a tendency to have less cross talk between the neighboring pixel. We assumed as the center point at the mid of the patch, the variations in the samples depend on the geometrical view of the patch as well as the noise level present in the sample.

It is assumed that reconstructed ocean surface image is a piecewise smooth surface in a cubical format [5]. To get the accurate piecewise smoothness of an image, it is considered the neighboring pixels present in the patch to get the maximum information of the reconstruction of the surface, a cubical model has been introduced shown in Fig. 5, which the patch contains samples and as well the neighboring samples. The size of the patch can be found by the mean and standard deviation. The cubical window size for each 
pixel is evaluated, which contains the samples in the patch. In order to evaluate the window size of each pixel, then,

$$
v_{j}=\frac{\sigma_{p j}}{\mu_{p j}}
$$

where $v_{j}$ is the ratio of variation of the samples located inside the patches which have the certain height and width. $p_{j}$ stands for $j^{\text {th }}$ patch, $\sigma_{p j}$ is the standard deviation of the samples, and $\mu_{p j}$ is the means. Our goal is to find the size of the patch. It needs to find the mean and standard deviation [5].

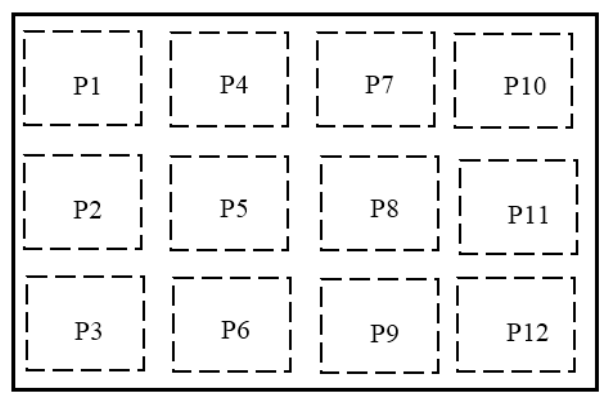

Fig. 5. Piecewise reconstruction.

Fig. 5 is the piecewise reconstruction of the patch which has lots of patches joined together piece wisely in order to have an accurate reconstruction of the ocean surface image. Each patch has the same geometry and dimension which we described earlier. As we used aperture filtering sampling the noise interference between pixels is minimized used this method.

\section{PIXEL RESOlution AND RECONSTRUCTION}

The pixel reconstruction is very essential parts of the reconstruction of images. In order to compute the value of the pixel the aperture filter is adopted, and some aliasing effect is deleted. There is an inverse proportional relationship the distance and weight of the sample because each sample contains noise in the sample within the patch of the defining cubical model, and weight is introduced in each sample that would enable us to reconstruct the pixels by evaluating the noise level in each sample. Hence the value of each pixel reconstruction has been done by convolving the kernel with a patch [5]. When in the reconstruction phase of pixel values, we need to determine the noise level present in each sample, let assume in our geometrical model as shown above where $D_{i}$ is the distance from the center point of the patch $c_{i j}$ and this distance varies according to the geometrical design of the patch, but in our case, the width and height assume to be constant. The method of the LIDAR reconstructed image is the piecewise smooth, when we reconstruct the signal, there is a possibility of the variation of the samples because of the geometry and there is some possibility that some samples contain noise within the patch, this noise between adjacent pixels is less due to aperture filter. As we get the smooth distance the less noise level present in the patch, similar idea has been developed in matching stereo vision [21].

$$
D_{i}=\left|Z-c_{i j}\right|
$$

where $D_{i}$ is the absolute distance between the centers $c_{i j}$ of the cubical and $Z$ is the noise present in the sample. The kernel smoothing technique is most widely used in medical image smoothing, in general, and it is simple to represent the weight sequence. In order to analysis the noise existed in the sample from an aperture filter by measuring the distance of the patch, the sample weight is defined in the reconstructed pixels.

$$
\emptyset\left(D_{i}\right) \propto \frac{1}{D_{i}}
$$

The sample weight corresponds to the weighting kernel $\varnothing\left(D_{i}\right)$ with variation in the scanning distance $D_{i}$, and it is to be a piecewise smooth. There are lots of methods to kernel smoothing, likewise, using cubical spline function, Multi quadratic spline function is adopted expressed as follows [5].

$$
\emptyset\left(D_{i}\right)=L \cdot \frac{(2 L-1) !}{(2 L) !} \beta^{2 k} \times Q
$$

The choice of parameters for smoothing the kernel is very critical selection of the accurate response, where parameter $\beta$ is constant, $2 L$ is the multi quadratic order, and $Q=\left(D_{i}^{2}\right) \frac{(2 L+1)}{2}$ is the parameter to control the smoothness of the kernel. The higher the value of the distance is, the more the smoothness of a kernel has.

\section{AdAPTIVE FILTER METHOD}

We discuss about the resolution problem occurred in the image and its methods to overcome the problem and to overcome the blurriness presented in the reconstructed image previous work has been done by implementing of the different image by estimation tools [21]. Assume $x(t)$ represent a reconstructed image at time $t$, it is the discrete time measurement, and assumes that ideal reconstructed image is a high-resolution image $y(t)$.

$$
x(t)=\mathrm{C} \cdot \mathrm{H}(t) y(t)+v(t)
$$

where $C$ is the decimation matrix with $\left[M^{2} \times N^{2}\right], M$ is the resolution of the reconstructed image $x(t), N$ is the resolution of the ideal image $y(t) . H(t)$ is the blur at the $t^{t h}$ with resolution $\left[N^{2} \times N^{2}\right]$ of the image. Our objective is to get the high resolution reconstructed image by using adaptive filtering method and as well as to minimize the blurness present in the reconstrued image to do, so we use adaptive filtering techniques we define here in matrix form, where. And $v(t)$ is the error present in the image at time $t$, it is assumed that random noise is present in the image, when we get the reconstructed image.

$$
y(t, j)=f(t, j) y(t)+s(t, j)
$$

where $f(t, j)$ is $\left[N^{2} \times N^{2}\right]$ matrix, $s(t, j)$ is the error, and random noise is assumed in image. Eq. (12) is an ideal image reconstructed at time $t$, and let the $x(t, j)$ is the reconstructed ocean surface image from the original an 
image $y(t)$, then combining Eq. (11), we get $N$ reconstructed images as follows,

$$
\begin{aligned}
& x(t, j)=C H(t, j) f(t, j) y(t)+E(t, j) \\
& \quad \text { For } 0 \leq j \leq N-1
\end{aligned}
$$

where $E(t, j)$ is the error of the reconstructed image and the original image at $t$. The LIDAR scanning at time $t$ and $N$ is the reconstructed image,

$$
E(t)=x(t, j)-C \cdot H(t, j) f(t, j) y(t)
$$

Eq. (14) is the model error, we need to minimize the reconstruction error by partial differentiate using Eq. (13).

\section{NOISE MODELING}

In this part, we need to estimate that how much noise adds to aperture filter samples, we reconstructed the image of the aperture filtered, which contains some noise. Noise model of the system is based on the assumptions. We have aperture filtered samples $S$ adds with zero- mean random noise $U$, and $S_{u}$ is the aperture filter samples which contained noisy samples, so that the noise model is formed like as follows,

$$
S_{u}=A S+U
$$

$A$ is the aperture function which is band limited that apply on the signal samples $S$ in Eq. (6), to get the better and high resolution of the reconstructed image. Now we are driving the estimation of the noise present in the sample by using Cramer Rao lower bound [22]. And have the minimum covariance for our estimation noise model, we developed the likelihood function as,

$$
S=A \sum_{i=1}^{N_{x}} \sum_{j=1}^{N_{y}}\left|k_{i, j}\right|^{-3 / 2} A_{i} B_{j}\left(\cos \alpha_{i}\right)\left(\sin \beta_{j}\right)+U
$$

when it will aperture filter sampling, we neglect constant parameter, because it is fixed parameter and we keep it constant so that it does not affect the signal $\left|k_{i, j}\right|^{-3 / 2}$ and the aperture coefficient $A$, we are driving the estimation of the noise present in the sample by using the Cramer Rao lower bound and it is widely used for estimation of the parameters [22]. $U$ is the noise. We are driving the CRLB form of our signal to estimate the noise contained by aperture filter.

$$
[F(S)]=\frac{1}{\sigma^{2}} \sum_{i=1}^{N_{x}} \sum_{j=1}^{N_{y}}\left(\frac{\partial S}{\partial i}\right)\left(\frac{\partial S}{\partial j}\right)
$$

Eq. (17) is the Fisher information matrix, we take a partial derivative of the signal when the scanning is performed in $i$ direction, similarly in $\mathrm{j}$-direction, respectively.

$$
\operatorname{Var}(S) \geq\left[F(S)^{-1}\right]
$$

In Eq. (18) the variance of the noise estimation of the aperture filtered samples $A S$ from the aperture filtered samples, which contain noise $S_{u}$ will be greater that the CRLB. We applied the scanning mechanism of the LIDAR from Fig. 3, to get the maximum information of the image. It has calculated that the optimal scanning angle $\Phi$ is $25^{\circ}$, it makes sure getting the maximum image scanning. It is found that the angle is selected less than $25^{\circ}$, it loses useful information of the image when we get reconstructed image, in addition selection of the scanning distance, is a critical choice, which is based on the original ocean surface image. The scanning distance is based on the size of the image, if we select, the less distance we cannot get the whole image information to be processed, and we may loss pixels information's to avoid that we choose the optimal scanning so the distance ranges are selected from 10 to 20 meters in the scanning range.

\section{Simulation Results}

In Fig. 6 we consider as an input photo images which has a boat on the ocean surface with the image size of 177 x 288 pixels, the ocean image dark image with image size of $360 \mathrm{x}$ 480 pixels, and the ocean surface sunlight image with size of $177 \times 288$ pixels respectively. We set the angle of the LIDAR scans $\Phi=25^{\circ}$ when we are performing the scanning in the both directions of the image.

Fig. 6 is the comparison of the proposed method of scanning of 5 and 7 with the method [1], the images of the ocean surface reflected by boat, dark light image and the sunlight effect have been taken as input images as shown in column (a). The reconstruction of the ocean surface images by using the method proposed [1] is in column (b) the reconstruction of image reflected by boat that the pixels and useful information have lost we cannot recover the whole images by using that method. We just recovered the some parts of the image, and useful pixel information is missing we just only found the some boundary pixel, as in the dark image we recover the some of the pixels information but blurriness present in the image, in addition when we reconstruct the sunlight image.

The reconstruction of ocean surface by scanning mechanism of 5 as shown in column (c), we set the angle $\Phi$ of the LIDAR scanning as $25^{\circ}$, this angle is the optimal parameter and performing one scanning of LIDAR and apply adaptive filter in order to get the reconstructed image. In column (c) is shown the enhanced reconstructed ocean surface image reflected by boat, by now performing the LIDAR scanning by increasing the range to 5 meters to cover the maximum parts of the image and applies adaptive filter, this image is better than the first image, we get a better resolution image and recover the maximum information. For dark image after 5 scanning meters, we achieved the good resolution of the reconstructed image compare with the method [1] less blurriness and clear image with mostly pixels have been recovered. In a sunlight image we cannot recover the image parts, and lost all information when we applied the method [1], but after applying scanning to the 5 meters, we reconstruct the some parts of the image successfully and get some useful information, but some pixels are still missing.

The reflected image by boat in column (d) as we increased the LIDAR scanning range from 5 to 7 meters, 
and apply the adaptive filter. It is found we achieved the high resolution reconstructed image comparatively with method [1] and as well as the scanning with the 5 , it is noted that as we increased the range of LIDAR scanning we get, the high resolution reconstructed image. Similarly for dark image we have been applied the same method now we get the better resolution reconstructed image, and recover the maximum pixel information, and for the sunlight image we achieved the good resolution image as compared to the method [1]. It is noticed that we recover the maximum information about the image, indeed by increasing the number of scanning distances will get the better reconstruction, but it also depends upon the sampling methods as well.
Input Image (a)
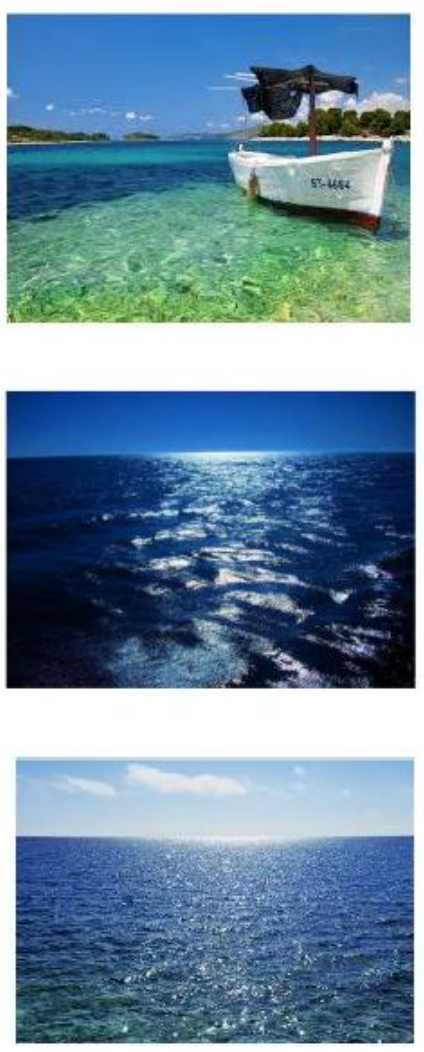

Result of $[1](b)$
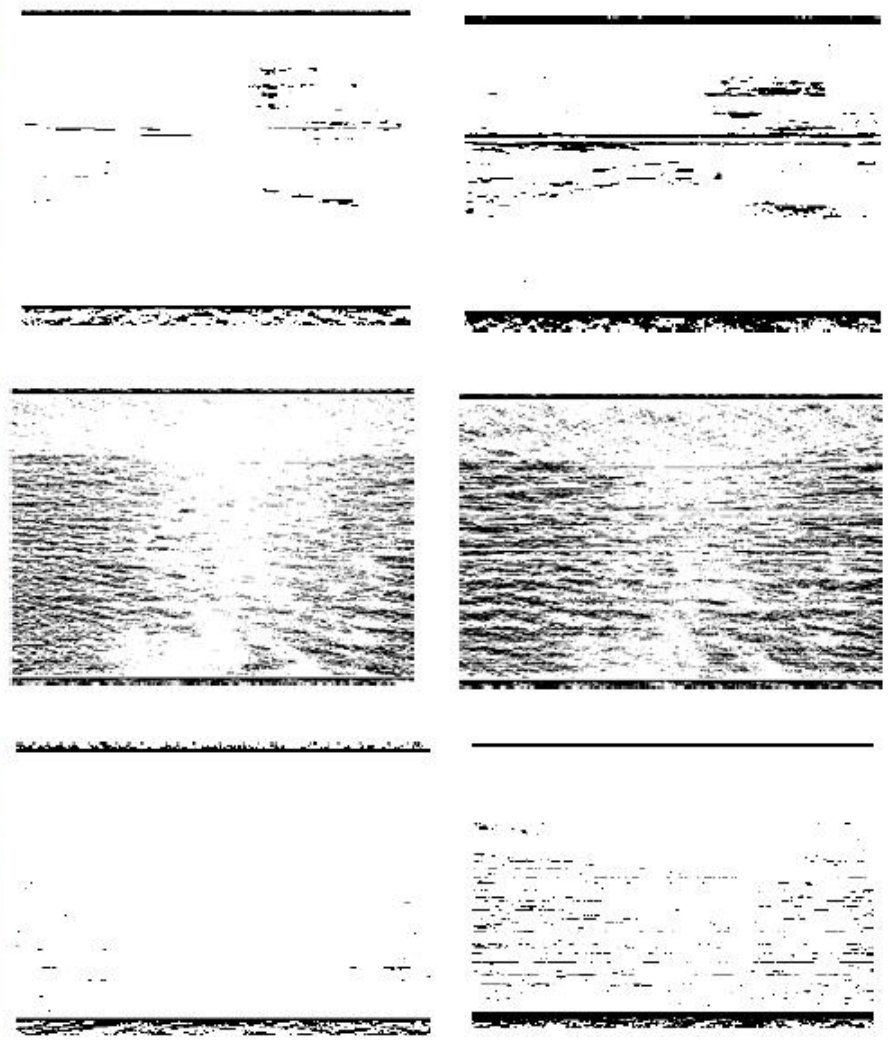

Fig. 6. Comparison of the proposed and method (1)
Improved Scanning 5 (c)
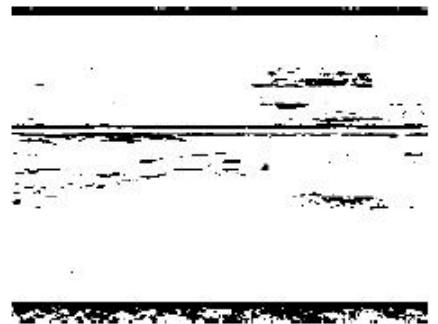

Improved Scanning 7 (d)

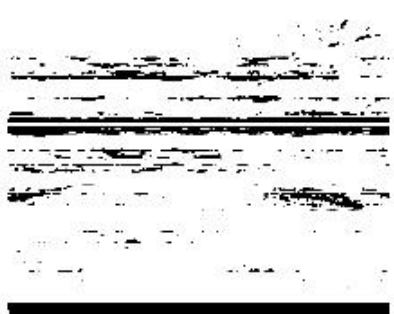

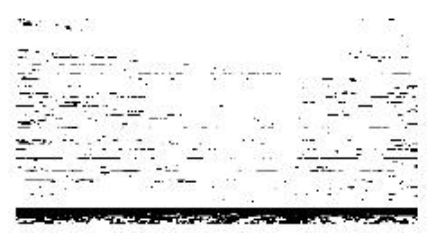

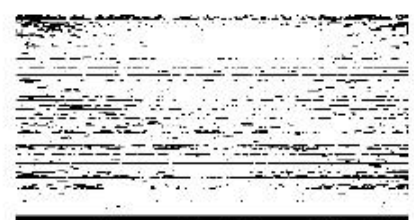

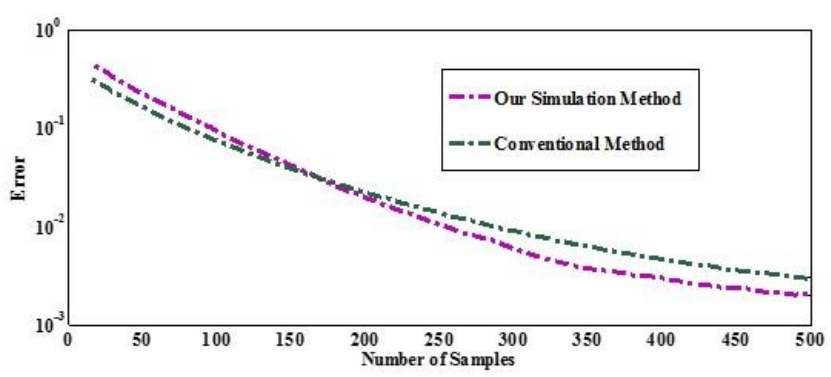

Fig. 7. Error comparison of our method with conventional method.

In order to evaluate the performance of reconstructing the high resolution ocean surface image, which is the graphical analysis between our method and the conventional method [1], we have performed 500 samples. We have defined the decimation matrix $C$ as constant and the variable $H$ is the blur matrix depended on the LIDAR scanning. From Fig. 7, it has obtained the aperture filter sample. Our sampling is based on the continuous signal construct from the axes coordinates of the image from the LIDAR scanning mechanism performed in two-dimension case. In Fig. 7, at the middle when sampling is nearly 175 samples, it is the critical zone, where the two graphs are overlapping, hence the error is also the same, once the samples have increased to 300 . The presented method error is less than the conventional method, and it is continuously less.

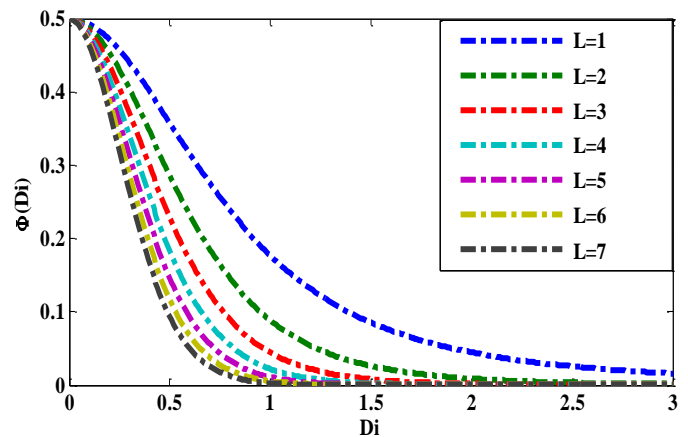

Fig. 8. Kernel behavior representation.

In Fig. 8, it is plotted the graphical representation of $\varnothing\left(D_{i}\right)$ with the distance according to Eq. (10) by defining the value of parameter $L$ in order to get the performance of the filter. The selections of $\beta$ and $L$ are very essential. This value does affect the performance of the kernel behaviors. The response of the filter is different when $L$ is equal to 1 . When it reaches to 3 the responses of the filter are identical, it is highlighted that the increasing the value of $L$ we get the smooth kernel response of the filter.

In Fig. 9 is demonstrated the comparison of our noise model with theoretical CRLB bound. The impact of the 
noise correlation has been evaluated of the signal which contained the aperture noisy sample, the response results shown that noise correlation is quite important, and the performance of the response is increasing, and the error has been decreasing shown is also less, the noise by using the aperture sampling method is significantly minimized to see Fig.9. We derive the CRLB of our noise signal model. The number of samples are taken as 50 , and $\sigma^{2}=0.5$.

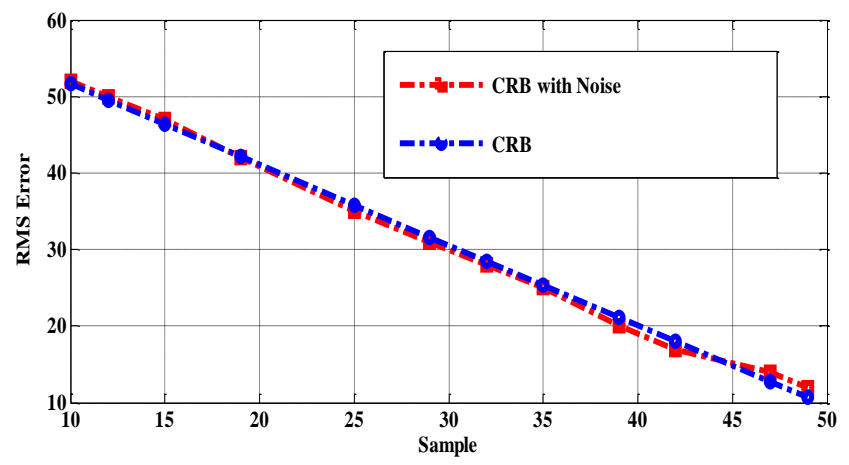

Fig. 9. Noise evaluation of aperture sample.

\section{CONCLUSIONS}

A new approached method has been presented to reconstruct the ocean surface image, which is based on the LIDAR scanning mechanism and sampling by aperture filter. To enhance the resolution of reconstructed image, we use an adaptive filter by defining the LIDAR scanning mechanism. The scheme, based on the scanning of the ocean surface in two-dimensional sampling. We have defined the cubical patch to reconstruct the pixel and as well the estimation of the noise present in the patch by measuring the distance. We have obtained the better piecewise reconstruction, and we have smoothed the kernel by defining the weight with distance assign the weighting kernel. The adaptive filter has been used for the image corresponding to the LIDAR scanning to get the best resolution image and to minimize the blurring present in the image. Our method has been presented better reconstruction of the ocean surface photo image and has obtained better resolution, and resolution gets better as we increase the scanning distance in addition it acquired less memory and computational time.

\section{ACKNOWLEDGMENT}

This work is supported by the National Natural Science Foundation of China (no. 61301211) and the Key Laboratory of Radar Imaging and Microwave Photonics, Ministry of Education, Nanjing University of Aeronautics and Astronautics, Nanjing, 210016, People Republic of China.

\section{REFERENCES}

[1] A. Muthuvel, M. Suhling, P. Hunziker, and M. Unser, "Variational image reconstruction from arbitrarily spaced samples a fast multiresolution spline solution," IEEE Trans. on Image Processing, vol. 14, no. 4, pp. 450-460, April 2005.

[2] T. Katsibas, T. Semertzidis, X. Lacondemine, and N.Grammalidis, "Signal processing for a laser based air data system in commercial aircrafts," in Proc. the IEEE Conference on Signal Processing Conference, 2008, pp.1-5, Lausanne (Switzerland).

[3] Light Detection and Ranging (LIDAR) Sensor Model, NGA Standardization documents, version 1.1. [Online]. Available:
http://www.gwg.nga.mil/focus_groups/csmwg/LIDAR_Formulation_ Paper_Version_1.1_110801.pdf

[4] A. Grigoryan, "Image reconstruction from finite number of projections: Method of transferring geometry," IEEE Trans. on Image Processing, vol. 22, no. 12, pp. 4738-4751, December 2013.

[5] M. Sarkis, "Adaptive Reconstruction of millimeter-wave radiometric images," IEEE Trans. on Image Processing, vol. 21, no. 9, pp. 41414151, September 2012

[6] H. Wenguang, H. Tingbo, W. Tao, B. Zhang, and L. Qixu, "Seasurface image super-resolution based on sparse representation," in Proc. IEEE International Conference of Image Analysis and Signal Processing (IASP), pp. 102-107, Hubei (China), 2011.

[7] M. Elad and A. Feuer, "Super resolution restoration of an image sequence adaptive filtering approach," IEEE Trans. on. Image Processing, vol. 8, no. 3, pp. 387-395, March 1999.

[8] Y. Jungang, J. Thompson, X. Huang, J. Tian, and Z. Zhimin, "Segmented reconstruction for compressed sensing SAR imaging," IEEE Trans. on Geosciences and Remote Sensing, vol. 51, pp. 4214-4225, July 2013.

[9] E. David and L. David, "Image reconstruction and enhanced resolution imaging from irregular samples," IEEE Trans. on Geoscience and Remote Sensing, vol. 39, no. 2, pp. 291-302, February 2001.

[10] F. Nouguier, G. Stephan, and C. Guerin, "Nonlinear ocean wave reconstruction algorithms based on simulated spatiotemporal data acquired by a flash LIDAR camera," IEEE Trans. on Geoscience and Remote Sensing, vol. 52, no. 3, pp. 1761-1771, March 2014

[11] W. A. Brent and D. Long, "Reconstruction from aperture-filtered samples with application to scatterometer image reconstruction," IEEE Trans. on Geo science and Remote Sensing, vol. 49, no. 5, pp. 1663-1676,May 2011.

[12] S. Kim, K. Nirmal, K. Bose, and H. Valenzuela, "Recursive reconstruction of high resolution image from noisy under sampled multi frames," IEEE Trans. on Acoustics, Speech and Signal Processing, vol. 38, no. 6 pp.1013-1027, June 1990.

[13] X. Xie, Y. Wenxing, and A. Entezari, "Bandlimited reconstruction of multidimensional images from irregular samples," IEEE Trans. on. Image Processing, vol. 22, no. 10, pp. 3950-3960, October 2013.

[14] A. F. Mansour and M. Gough, "Processing intensive full-waveform aerial laser scanning Matlab jobs through condor," Internet of Things and Cloud Computing, vol. 1, no.1, pp. 5-14, 2013.

[15] B. Vandeghinste, B. Goossens, R. Holen, C. Vanhove, A. Pizurica, S Vandenberghe, and S. Staelens, "Iterative CT reconstruction using shearlet-based regularization," IEEE Trans. on Nuclear Science, vol. 60 , no. 5 , October 2013

[16] C. G. Holsbach and J. Bermudez, "Statistical analysis of the LMS algorithm applied to super-resolution image reconstruction," IEEE Trans. on Signal Processing, vol. 55, no. 5, pp. 2084-2095,May 2007.

[17] H. Simon, M. Kleinsteuber, and K. Diepold, "Analysis operator learning and its application to image reconstruction," IEEE Trans. on Image Processing, vol. 22, no. 6, pp. 2138-2150,June 2013.

[18] S. Motoyuki, K. Takahashi, and L. Yi, "Optimization of data sampling and image reconstruction by GPR," in Proc. IEEE International Conference on GPR, 2014, pp. 615-618, Brussels, Belgium.

[19] C. Tang, S. Avidan, and W. Freeman, "The patch transform," IEEE Trans. on Pattern Analysis and Machine Intelligence, vol. 32, pp. 1489-501, August 2010.

[20] C. Tang, M. Butman, S. Avidan, and W. Freeman, "The patch transform and its applications to image editing," in Proc. IEEE Conference on Computer Vision and Pattern Recognition (CPVR), pp. 1-8, Anchorage, AK, 2008.

[21] M. Elad and A. Feuer, "Super resolution reconstruction of an image," IEEE Electrical and Electronics Engineer, pp. 391-394, Jerusalem (Israel), 1996.

[22] A. Jibia, U. M. Salami, O. Khalifa, and F .Elfaki, "Cramer-Rao lower bound for parameter estimation of multi exponential signals," in Proc. IEEE Systems, Signals and Image Processing, Conference, pp. 1-5. Chalkida, Greece, 2009.

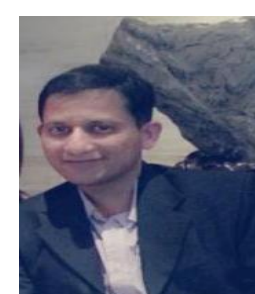

Muhammad Sameer Sheikh was born in Karach Pakistan. He received his bachelor degree in electronic engineering from Hamdard University, Karachi, Pakistan in 2007. He received his master degree in telecommunication engineering from Hamdard University Karachi, Pakistan in 2010. $\mathrm{He}$ is currently a $\mathrm{PhD}$ candidate at College of Electronics and Information Engineering, Nanjing University of Aeronautics and Astronautics. 
Nanjing, China. His main research interest focused on the area of computational electromagnetics, radar signal processing, image processing and digital signal processing.

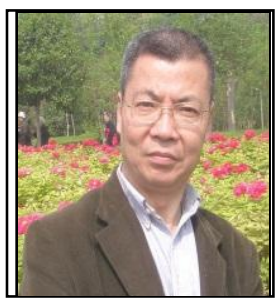

Qunsheng Cao received his $\mathrm{Ph} . \mathrm{D}$. in electrica engineering from the Hong Kong Polytechnic University in 2001. From 2001 to 2005 he worked as a research associate in the Department of Electrical Engineering, University of Illinois

at Urbana-Champaign and at the Army High Performance Computing Research Center (AHPCRC), University of Minnesota. In 2006, Dr. Cao joined the Nanjing University of Aeronautics and Astronautics (NUAA), China, as a professor of electrical engineering. Dr. Cao's current research interests are in computational electromagnetics, antenna and microwave technology and the radar signal processing. Dr. Cao has published more than 120 academic papers in refereed journals and conference proceedings.

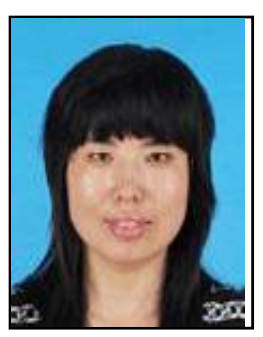

Caiyun Wang was born in Shanxi, China, on September 30, 1975. She graduated in 1996 with a B.S. degree and in 1999 with a M.S. degree. She received the $\mathrm{Ph} . \mathrm{D}$. degree in signal and information processing from Beihang University, Beijing, China, in 2008. She is currently an associate professor with the College of Astronautics, Nanjing University of Aeronautics and Astronautics (NUAA). Her major research interests are in the fields of radar automatic target recognition (RATA), radar signal processing, image processing, digital signal processing and compress sensing. 